\title{
A family of singular ordinary differential equations of the third order with an integral boundary condition
}

\author{
Mahdi Boukrouche $e^{1}$ and Domingo A. Tarzia ${ }^{2 *}$
}

\section{"Correspondence:}

DTarzia@austral.edu.ar

${ }^{2}$ Departamento de

Matemática-CONICET, FCE, Univ.

Austral, Rosario, Argentina

Full list of author information is

available at the end of the article (c) The Author(s) 2018. This article is distributed under the terms of the Creative Commons Attribution 4.0 International License (http://creativecommons.org/licenses/by/4.0/), which permits unrestricted use, distribution, and reproduction in any medium, provided you give appropriate credit to the original author(s) and the source, provide a link to the Creative Commons license, and indicate if changes were made.

\begin{abstract}
We establish in this paper the equivalence between a Volterra integral equation of the second kind and a singular ordinary differential equation of the third order with two initial conditions and an integral boundary condition, with a real parameter. This equivalence allows us to obtain the solution to some problems for non-classical heat equation, the continuous dependence of the solution with respect to the parameter and the corresponding explicit solution to the considered problem.
\end{abstract}

MSC: 34A05; 34B10; 34B16; 35C15; 35K05; 35K20; 45D05; 45E10

Keywords: Singular ordinary differential equation of third order; Integral boundary condition; Volterra integral equation; Explicit solution; Non-classical heat equation

\section{Introduction}

We consider the family of singular ordinary differential equations of the third order with an integral boundary condition, indexed by a parameter $\lambda \in \mathbb{R}$ given by

$$
\left.\begin{array}{lll}
y^{(3)}(t)-\lambda^{2} y(t)=\frac{\lambda}{2 \sqrt{\pi}} \frac{1}{t^{3 / 2}}, & 0<t<1, \\
y(0)=1, & y^{\prime}(0)=0, & y^{(2)}(1)=-\frac{\lambda}{\sqrt{\pi}}+\lambda^{2} \int_{0}^{1} y(t) d t,
\end{array}\right\}
$$

where $y^{(n)}$ denotes the $n$-derivative of the function $y$.

Singular boundary value problems arise very frequently in fluid mechanics and in other branches of applied mathematics. There are results on the existence and asymptotic estimates of solutions for third order ordinary differential equations with singularly perturbed boundary value problems, which depend on a small positive parameter, see, for example [1-3], on third order ordinary differential equations with singularly perturbed boundary value problems and with nonlinear coefficients or boundary conditions, see for example [4-7], on third order ordinary differential equations with nonlinear boundary value problems, see for example [8,9], on existence results for third order ordinary differential equations, see for example [10-12], and particularly third order ordinary differential equations with integral boundary conditions, see for example [13-21].

In the last years there have been published several papers which consider integral or nonlocal boundary conditions on different branches of applications, e.g., for the heat equations, see for example [22-32], for the wave equations [33], for the second order ordinary 
differential equations, see for example [34-40], for the fourth order ordinary differential equations, see for example [41, 42], for higher order ordinary differential equations, see for example [43], for fractional differential equations, see for example [44-46].

Our goal is to prove in Sect. 2 that the system (1.1) is equivalent to the following Volterra integral equation of the second kind:

$$
y(t)=1-\frac{2 \lambda}{\sqrt{\pi}} \int_{0}^{t} y(\tau) \sqrt{t-\tau} d \tau, \quad 0<t<1(\lambda \in \mathbb{R})
$$

which allows us to obtain the solution to some problems for non-classical heat equation for any real parameter $\lambda$ (see [47-53]).

We remark that the Volterra integral equation (1.2) can also be considered and extended for $t>0$, that is,

$$
y(t)=1-\frac{2 \lambda}{\sqrt{\pi}} \int_{0}^{t} y(\tau) \sqrt{t-\tau} d \tau, \quad t>0(\lambda \in \mathbb{R}) .
$$

In Sect. 3, we establish the dependence of the family of boundary value problems for singular ordinary differential equations of third order (1.1) with respect to the parameter $\lambda \in \mathbb{R}$ by using the equivalence with the Volterra integral equation (1.2).

\section{Equivalence and existence results}

Preliminarily, we give some results useful in the next sections.

Lemma 2.1 We have the following properties for all $t>0$ :

$$
\begin{aligned}
& \int_{0}^{t}\left(\int_{0}^{\tau} y(\xi) d \xi\right) d \tau=\int_{0}^{t} y(\tau)(t-\tau) d \tau \\
& \int_{0}^{t}\left(\int_{0}^{\xi} y(\tau)(t-\tau) d \tau\right) d \xi=\int_{0}^{t} y(\tau)(t-\tau)^{2} d \tau \\
& \int_{0}^{t} \frac{y(\tau)}{\sqrt{t-\tau}} d \tau=2 \sqrt{t}+2 \int_{0}^{t} y^{\prime}(\tau) \sqrt{t-\tau} d \tau \\
& \int_{\sigma}^{t} \frac{\sqrt{\tau-\sigma}}{\sqrt{t-\tau}} d \tau=\frac{\pi}{2}(t-\sigma), \\
& \int_{\sigma}^{t} \frac{d \tau}{\sqrt{t-\tau} \sqrt{\tau-\sigma}}=\pi .
\end{aligned}
$$

Proof The first three properties (2.1)-(2.3) follow from the simple integration process. To prove (2.4), we use the change of variable $\tau=\sigma+(t-\sigma) \xi$ then we obtain

$$
\begin{aligned}
\int_{\sigma}^{t} \frac{\sqrt{\tau-\sigma}}{\sqrt{t-\tau}} d \tau & =(t-\sigma) \int_{0}^{1} \sqrt{\frac{\xi}{1-\xi}} d \xi=(t-\sigma) \int_{0}^{1} \xi^{\frac{3}{2}-1}(1-\xi)^{\frac{1}{2}-1} d \xi \\
& =(t-\sigma) B\left(\frac{3}{2}, \frac{1}{2}\right)=(t-\sigma) \frac{\Gamma\left(\frac{3}{2}\right) \Gamma\left(\left(\frac{1}{2}\right)\right.}{\Gamma(2)}=\frac{\pi}{2}(t-\sigma),
\end{aligned}
$$


where $B$ and $\Gamma$ are the known Beta and Gamma functions defined by

$$
\begin{aligned}
& B(x, y)=\int_{0}^{1} t^{x-1}(1-t)^{y-1} d t, \quad x>0, y>0, \\
& \Gamma(x)=\int_{0}^{+\infty} t^{x-1} e^{-t} d t, \quad x>0
\end{aligned}
$$

with the well-known relations

$$
\begin{aligned}
& B(x, y)=\frac{\Gamma(x) \Gamma(y)}{\Gamma(x+y)}, \quad \Gamma(x+1)=x \Gamma(x) \quad \forall x>0, \\
& \Gamma\left(\frac{1}{2}\right)=\sqrt{\pi}, \quad \Gamma(n+1)=n ! \quad \forall n \in \mathbb{N} .
\end{aligned}
$$

To prove (2.5) we use the same change of variable, so we obtain

$$
\int_{\sigma}^{t} \frac{d \tau}{\sqrt{t-\tau} \sqrt{\tau-\sigma}}=\int_{0}^{1} \frac{d \xi}{\sqrt{\xi(1-\xi)}}=B\left(\frac{1}{2}, \frac{1}{2}\right)=\frac{\Gamma\left(\frac{1}{2}\right) \Gamma\left(\frac{1}{2}\right)}{\Gamma(1)}=\pi
$$

Theorem 2.2 $y$ is a solution to the singular boundary value problems (1.1) if and only if $y$ is a solution to the Volterra integral equation (1.2) for any real parameter $\lambda>0$.

Proof Firstly, we consider that $y$ is a solution to the singular boundary value problems (1.1). Then, by using an integration in variable $t$ we obtain

$$
y^{(2)}(t)=y^{(2)}(0)+\lambda^{2} \int_{0}^{t} y(\tau) d \tau-\frac{\lambda}{\sqrt{\pi t}}, \quad 0<t<1
$$

thus

$$
y^{(2)}(1)=y^{(2)}(0)+\lambda^{2} \int_{0}^{1} y(\tau) d \tau-\frac{\lambda}{\sqrt{\pi}}
$$

And using the integral boundary condition we get

$$
y^{(2)}(1)=-\frac{\lambda}{\sqrt{\pi}}+\lambda^{2} \int_{0}^{1} y(t) d t
$$

so $y^{(2)}(0)=0$. Thus taking this new condition into account, from (2.6) by using an integration in variable $t$, the condition $y^{\prime}(0)=0$ and $(2.1)$ we get

$$
\begin{aligned}
y^{\prime}(t) & =\lambda^{2} \int_{0}^{t}\left(\int_{0}^{\tau} y(\sigma) d \sigma\right) d \tau-\frac{2 \lambda \sqrt{t}}{\sqrt{\pi}} \\
& =\lambda^{2} \int_{0}^{t} y(\tau)(t-\tau) d \tau-\frac{2 \lambda \sqrt{t}}{\sqrt{\pi}}, \quad 0<t<1 .
\end{aligned}
$$


Finally, from (2.7) by using another integration in the variable $t$, and the condition $y(0)=1$, we obtain

$$
\begin{aligned}
y(t) & =1+\lambda^{2} \int_{0}^{t}\left(\int_{0}^{\tau} y(\sigma)(\tau-\sigma) d \sigma\right) d \tau-\frac{4 \lambda 3}{3 \sqrt{\pi}} t^{3 / 2} \\
& =1+\lambda^{2} \int_{0}^{t} y(\tau)(t-\tau)^{2} d \tau-\frac{4 \lambda}{3 \sqrt{\pi}} t^{3 / 2}, \quad 0<t<1 .
\end{aligned}
$$

We cannot arrive directly at the Volterra equation (1.2), but we can define the auxiliary function

$$
\varphi(t)=y(t)-1+\frac{2 \lambda}{\sqrt{\pi}} \int_{0}^{t} y(\tau) \sqrt{t-\tau} d \tau
$$

and now our goal is to prove that $\varphi=0$. We have $\varphi(0)=0$, by using the boundary $y(0)=1$.

Now, we compute the first derivative of $\varphi$ using the property (2.3), we get

$$
\begin{aligned}
\varphi^{\prime}(t) & =y^{\prime}(t)+\frac{\lambda}{\sqrt{\pi}} \int_{0}^{t} \frac{y(\tau)}{\sqrt{t-\tau}} d \tau \\
& =y^{\prime}(t)+\frac{\lambda}{\sqrt{\pi}}\left(2 \sqrt{t}+2 \int_{0}^{t} y^{\prime}(\tau) \sqrt{t-\tau} d \tau\right), \quad 0<t<1 .
\end{aligned}
$$

On the other hand, by using (2.9), (2.7), (2.10) and the property (2.4) we obtain

$$
\begin{aligned}
\int_{0}^{t} \frac{\varphi(\tau)}{\sqrt{t-\tau}} d \tau & =\int_{0}^{t} \frac{y(\tau)}{\sqrt{t-\tau}} d \tau-2 \sqrt{t}+\frac{2 \lambda}{\sqrt{\pi}} \int_{0}^{t} \frac{\int_{0}^{\tau} y(\sigma) \sqrt{\tau-\sigma} d \sigma}{\sqrt{t-\tau}} d \tau \\
& =\int_{0}^{t} \frac{y(\tau)}{\sqrt{t-\tau}} d \tau-2 \sqrt{t}+\lambda \sqrt{\pi} \int_{0}^{t} y(\tau)(t-\tau) d \tau \\
& =\int_{0}^{t} \frac{y(\tau)}{\sqrt{t-\tau}} d \tau-2 \sqrt{t}+\lambda \sqrt{\pi}\left(\frac{y^{\prime}(t)}{\lambda^{2}}+\frac{2}{\lambda \sqrt{\pi}} \sqrt{t}\right) \\
& =\int_{0}^{t} \frac{y(\tau)}{\sqrt{t-\tau}} d \tau+\frac{\sqrt{\pi}}{\lambda} y^{\prime}(t)=\frac{\sqrt{\pi}}{\lambda} \varphi^{\prime}(t), \quad 0<t<1 .
\end{aligned}
$$

That is,

$$
\varphi^{\prime}(t)=\frac{\lambda}{\sqrt{\pi}} \int_{0}^{t} \frac{\varphi(\tau)}{\sqrt{t-\tau}} d \tau, \quad 0<t<1
$$

thus $\varphi^{\prime}(0)=0$. Therefore, we have

$$
\varphi^{\prime}(t)=\frac{\lambda}{\sqrt{\pi}} \int_{0}^{t} \frac{\varphi(t-\tau)}{\sqrt{\tau}} d \tau, \quad 0<t<1,
$$

and then we obtain

$$
\varphi^{(2)}(t)=\frac{\lambda}{\sqrt{\pi}} \int_{0}^{t} \frac{\varphi^{\prime}(t-\tau)}{\sqrt{\tau}} d \tau=\frac{\lambda}{\sqrt{\pi}} \int_{0}^{t} \frac{\varphi^{\prime}(\tau)}{\sqrt{t-\tau}} d \tau, \quad 0<t<1,
$$

thus $\varphi^{(2)}(0)=0$, and so on we obtain $\varphi^{(n)}(0)=0$ for all $n \in \mathbb{N}$, then this part holds. 
Secondly, we consider that $y$ is a solution of the Volterra integral equation (1.2), then we have the condition $y(0)=1$, which is automatically satisfied.

Then, by derivation of (1.2) and by using the property (2.4) we have

$$
\begin{aligned}
y^{\prime}(t) & =-\frac{\lambda}{\sqrt{\pi}} \int_{0}^{t} \frac{y(\tau)}{\sqrt{t-\tau}} d \tau \\
& =-\frac{\lambda}{\sqrt{\pi}}\left(2 \sqrt{t}-\frac{2 \lambda}{\sqrt{\pi}}\left(\int_{0}^{t} \frac{1}{\sqrt{t-\tau}}\left(\int_{0}^{\tau} y(\sigma) \sqrt{\tau-\sigma} d \sigma\right) d \tau\right)\right) \\
& =-\frac{2 \lambda}{\sqrt{\pi}} \sqrt{t}+\frac{2 \lambda^{2}}{\pi} \int_{0}^{t}\left(\int_{\sigma}^{t} \frac{\sqrt{\tau-\sigma}}{\sqrt{t-\tau}} d \tau\right) y(\sigma) d \sigma \\
& =-\frac{2 \lambda}{\sqrt{\pi}} \sqrt{t}+\lambda^{2} \int_{0}^{t} y(\sigma)(t-\sigma) d \sigma, \quad 0<t<1,
\end{aligned}
$$

and the boundary condition $y^{\prime}(0)=0$ holds. Therefore, from (2.14) we have

$$
y^{(2)}(t)=-\frac{\lambda}{\sqrt{\pi t}}+\lambda^{2} \int_{0}^{t} y(\tau) d \tau, \quad 0<t<1
$$

thus for $t=1$ we get the integral boundary condition.

Finally, from (2.15) we have

$$
y^{(3)}(t)=\frac{\lambda}{\sqrt{\pi}} t^{-3 / 2}+\lambda^{2} y(t), \quad 0<t<1,
$$

so the singular boundary value problems (1.1) holds for any real parameter $\lambda>0$, thus the proof of the theorem is complete.

It is well known that there exists a unique solution of the Volterra integral equation (1.3), that is, the Volterra integral equation (1.2) extended for $t>0$; see [49, 54]. Now, we will find the explicit solution of the Volterra integral equation (1.3).

Theorem 2.3 The solution of the Volterra integral equation (1.3) is given by the following expression:

$$
y(t)=I(t)-\sqrt{\frac{2}{\pi}} J(t), \quad t>0,
$$

with

$$
\begin{aligned}
& I(t)=\sum_{n=0}^{+\infty} \frac{\left(\lambda^{2 / 3} t\right)^{3 n}}{(3 n) !}, \\
& J(t)=\sum_{n=0}^{+\infty} \frac{\left(2 \lambda^{2 / 3} t\right)^{\frac{3(2 n+1)}{2}}}{(3(2 n+1)) ! !},
\end{aligned}
$$

being series with infinite radii of convergence and we use the definition

$$
(2 n+1) ! !=(2 n+1)(2 n-1)(2 n-3) \cdots 5 \cdot 3 \cdot 1 .
$$

for the compactness expression. 
Proof By using the Adomian method $[55,56]$ we propose, for the solution of the Volterra integral equation (1.3), the series of expansion functions given by

$$
y(t)=\sum_{n=0}^{+\infty} y_{n}(t)
$$

and we obtain the following recurrence expansions:

$$
y_{0}(t)=1, \quad y_{n}(t)=-\frac{2 \lambda}{\sqrt{\pi}} \int_{0}^{t} y_{n-1}(\tau) \sqrt{t-\tau} d \tau, \quad \forall n \geq 1 .
$$

Then, following [49], we obtain (2.17) where $I(t)$ and $J(t)$ are given by (2.18) and (2.19), respectively, and the result holds.

The solution of the Volterra integral equation (1.3) is the key for obtaining the solution of the non-classical heat conduction problem given by

$$
\begin{aligned}
& u_{t}(x, t)-u_{x x}(x, t)=-\lambda \int_{0}^{t} u_{x}(0, \tau) d \tau, \quad x>0, t>0, \\
& u(0, t)=0, \quad t>0, \\
& u(x, 0)=h_{0}>0, \quad x>0,
\end{aligned}
$$

with a parameter $\lambda \in \mathbb{R}$. Then the solution of the problem above is given by

$$
u(x, t)=h_{0} \operatorname{erf}\left(\frac{x}{2 \sqrt{t}}\right)-\lambda \int_{0}^{t} \operatorname{erf}\left(\frac{x}{2 \sqrt{t-\tau}}\right) U(\tau) d \tau,
$$

where $U(t)$ is given by

$$
U(t)=\frac{h_{0}}{\sqrt{\pi}} \int_{0}^{t} \frac{g(\tau)}{\sqrt{t-\tau}} d \tau
$$

and $g$ is the solution of the Volterra integral equation (1.3). Moreover, the heat flux on $x=0$ is given by

$$
u_{x}(0, t)=U^{\prime}(t)=\frac{h_{0}}{\sqrt{\pi t}}-h_{0} \lambda \int_{0}^{t} g(\tau) d \tau, \quad t>0 .
$$

For the complete proof see [49].

\section{Dependence of the solution with respect to $\lambda$}

From now on, we will consider that the solution to the singular ordinary differential equation of the third order with an integral boundary condition (1.1) or equivalently the solution of the Volterra integral equation (1.2) depends also on the parameter $\lambda \in \mathbb{R}$. From now on, without loss of generality, we will consider the Volterra integral equation (1.3)

We consider that $t \mapsto g_{\lambda}(t)$ be the solution of the Volterra integral equation (1.2) for the parameter $\lambda$. For $\varepsilon \in(0,1)$ be a fixed real number and $T>0$, let consider the parameter $\lambda$ such that

$$
|\lambda| \leq \lambda_{\varepsilon, T}=\frac{3 \sqrt{\pi}}{4} \frac{\varepsilon}{T^{3 / 2}}
$$


and we define the norm

$$
\|g\|_{T}=\max _{0 \leq t \leq T}|g(t)| .
$$

Therefore, we obtain the following dependence results.

Theorem 3.1 We have the boundedness:

$$
\left\|g_{\lambda}\right\|_{T} \leq \frac{1}{1-\varepsilon}, \quad \forall \lambda:|\lambda| \leq \lambda_{\varepsilon, T}
$$

Moreover, the application $\lambda \mapsto g_{\lambda}(t)$ defined from $\left[-\lambda_{\varepsilon, T}, \lambda_{\varepsilon, T}\right]$, to $\mathcal{C}([0, T))$ is Lipschitzian.

Proof From the Volterra integral equation (1.2) we obtain

$$
\begin{aligned}
\left|g_{\lambda}(t)\right| & \leq 1+\frac{2|\lambda|}{\sqrt{\pi}}\left\|g_{\lambda}\right\|_{t} \int_{0}^{t} \sqrt{t-\tau} d \tau \\
& \leq 1+\frac{4}{3 \sqrt{\pi}} \lambda_{\varepsilon, T} T^{3 / 2}\left\|g_{\lambda}\right\|_{T}
\end{aligned}
$$

and by using (3.1) follows (3.2). Moreover, consider $g_{i}(t)$ the solution of the Volterra integral equation (1.2) for $\lambda_{i}(i=1,2)$, such that

$$
\left|\lambda_{i}\right| \leq \lambda_{\varepsilon, T}
$$

Then we have

$$
\begin{aligned}
\left|g_{2}(t)-g_{1}(t)\right| & \leq \frac{2}{\sqrt{\pi}}\left|\lambda_{2}-\lambda_{1}\right|\left\|g_{1}\right\|_{t} \int_{0}^{t} \sqrt{t-\tau} d \tau+\frac{2\left|\lambda_{2}\right|}{\sqrt{\pi}}\left\|g_{1}-g_{2}\right\|_{t} \int_{0}^{t} \sqrt{t-\tau} d \tau \\
& \leq \frac{4 T^{3 / 2}}{3 \sqrt{\pi}}\left[\left|\lambda_{2}-\lambda_{1}\right|\left\|g_{1}\right\|_{T}+\left|\lambda_{2}\right|\left\|g_{2}-g_{1}\right\|_{T}\right] .
\end{aligned}
$$

Therefore, we get

$$
\left\|g_{2}-g_{1}\right\|_{T} \leq \frac{4}{3 \sqrt{\pi}} \frac{T^{3 / 2}}{(1-\varepsilon)^{2}}\left|\lambda_{2}-\lambda_{1}\right|
$$

thus the result holds.

Now, we obtain the dependence of the solution to the non-classical heat conduction problem (2.20)-(2.22) with respect to the parameter $\lambda$. We consider that $U_{\lambda}$ and $u_{\lambda}$ are given, respectively, by

$$
U_{\lambda}(t)=\frac{h_{0}}{\sqrt{\pi}} \int_{0}^{t} \frac{g_{\lambda}(\tau)}{\sqrt{t-\tau}} d \tau
$$

and

$$
u_{\lambda}(x, t)=h_{0} \operatorname{erf}\left(\frac{x}{2 \sqrt{t}}\right)-\lambda \int_{0}^{t} \operatorname{erf}\left(\frac{x}{2 \sqrt{t-\tau}}\right) U_{\lambda}(\tau) d \tau .
$$

Then we obtain the following results. 
Theorem 3.2 We have the boundedness:

$$
\left\|U_{\lambda}\right\|_{T} \leq \frac{2 h_{0}}{\sqrt{\pi}} \frac{T^{1 / 2}}{1-\varepsilon}, \quad \forall \lambda:|\lambda| \leq \lambda_{\varepsilon, T}
$$

Moreover, the application $\lambda \mapsto U_{\lambda}(t)$, from $\left[-\lambda_{\varepsilon, T}, \lambda_{\varepsilon, T}\right]$ to $\mathcal{C}([0, T])$ is Lipschitzian. We have also the following boundedness:

$$
\left\|u_{\lambda}\right\|_{[0,+\infty[\times[0, T]} \leq h_{0}\left(1+\frac{3 \varepsilon}{2(1-\varepsilon)}\right) \quad \forall \lambda:|\lambda| \leq \lambda_{\varepsilon, T}
$$

the estimates

$$
\left\|u_{\lambda}-u_{0}\right\|_{[0,+\infty[\times[0, T]} \leq \frac{2 h_{0}}{\sqrt{\pi}} \frac{T^{3 / 2}}{1-\varepsilon}|\lambda|, \quad \forall \lambda:|\lambda| \leq \lambda_{\varepsilon, T},
$$

and find that the application $\lambda \mapsto u_{\lambda}(x, t)$, from $\left[-\lambda_{\varepsilon, T}, \lambda_{\varepsilon, T}\right]$ to $\mathcal{C}([0,+\infty[\times[0, T])$ is Lipschitzian.

Proof From (2.24), we have

$$
\left|U_{\lambda}(t)\right| \leq \frac{h_{0}}{\sqrt{\pi}}\left\|g_{\lambda}\right\|_{t} \int_{0}^{t} \frac{d \tau}{\sqrt{t-\tau}} \leq \frac{2 h_{0}}{\sqrt{\pi}} \frac{T^{1 / 2}}{1-\varepsilon}
$$

thus (3.6) holds. Consider now $U_{i}(t)$ given by (3.4), for $\lambda_{i}(i=1,2)$ satisfying $\left|\lambda_{i}\right| \leq \lambda_{\varepsilon, T}$. We have

$$
\left|U_{2}(t)-U_{1}(t)\right| \leq \frac{2 h_{0} T^{1 / 2}}{\sqrt{\pi}}\left\|g_{2}-g_{1}\right\|_{T} \leq \frac{8 h_{0} T^{2}}{3 \pi(1-\varepsilon)^{2}}\left|\lambda_{2}-\lambda_{1}\right|
$$

thus the application $\lambda \mapsto U_{\lambda}$ is Lipschitzian.

From (3.5), we have

$$
\left|u_{\lambda}(x, t)\right| \leq h_{0}+t|\lambda|\left\|U_{\lambda}\right\|_{t} \leq h_{0}\left(1+\frac{3 \varepsilon}{2(1-\varepsilon)}\right), \quad \forall x \in[0,+\infty[, \forall t \in[0, T],
$$

thus (3.7) holds.

From (3.5) also, we have

$$
\left|u_{\lambda}(x, t)-u_{0}(x, t)\right| \leq t|\lambda||| U_{\lambda} \|_{t} \leq \frac{2 h_{0}}{\sqrt{\pi}} \frac{T^{3 / 2}}{1-\varepsilon}|\lambda|
$$

thus (3.8) holds.

Consider now $u_{i}(x, t)$ given by (3.5) for $\lambda_{i}(i=1,2)$ satisfying $\left|\lambda_{i}\right| \leq \lambda_{\varepsilon, T}$. Then we have

$$
\begin{aligned}
\left|u_{2}(x, t)-u_{1}(x, t)\right| & \leq t\left|\lambda_{2}-\lambda_{1}\right|\left\|U_{1}\right\|_{t}+t\left|\lambda_{2}\right|\left\|U_{2}-U_{1}\right\|_{t} \\
& \leq T\left|\lambda_{2}-\lambda_{1}\right|\left\|U_{1}\right\|_{T}+T\left|\lambda_{2}\right|\left\|U_{2}-U_{1}\right\|_{T} \\
& \leq \frac{2 h_{0} T^{1 / 2}}{\sqrt{\pi}(1-\varepsilon)}\left(T+\frac{\varepsilon \pi}{1-\varepsilon}\right)\left|\lambda_{2}-\lambda_{1}\right| \quad \forall x \in[0,+\infty[, \forall t \in[0, T],
\end{aligned}
$$


thus

$$
\left\|u_{2}-u_{1}\right\|_{[0,+\infty[\times[0, T]} \leq \frac{2 h_{0} T^{1 / 2}}{\sqrt{\pi}(1-\varepsilon)}\left(\frac{\varepsilon \pi}{1-\varepsilon}+T\right)\left|\lambda_{2}-\lambda_{1}\right|
$$

and the result holds.

Conclusion We have obtained the equivalence between a family of singular ordinary differential equations of the third order with two initial conditions and an integral boundary condition (1.1), and the Volterra integral equation (1.2) with a parameter $\lambda \in \mathbb{R}$. We have also given the explicit solution of these equations which can be extended for all $t>0$, and then some non-classical heat conduction problems can be solved explicitly, for any real parameter $\lambda$. Finally, we have established the dependence of the family of singular boundary problems of the third order with respect to the parameter $\lambda$.

\begin{abstract}
Acknowledgements
This paper was partially sponsored by the Institut Camille Jordan St-Etienne University for first author, and the projects PIP \# 0275 from CONICET-Austral and ANPCyT PICTO Austral 2016 \# 090 (Rosario, Argentina), and Grant AFOSR-SOARD FA 9550-14-1-0122 for the second author. The authors thank three anonymous reviewers whose comments helped them to improve our paper.
\end{abstract}

\title{
Competing interests
}

The authors declare that there are no conflict of interest regarding the publication of this paper.

\section{Authors' contributions}

The authors contributed equally to the writing of this paper. The authors read and approved the final manuscript.

\section{Author details}

${ }^{1}$ Institut Camille Jordan CNRS UMR 5208, Lyon University, Saint-Etienne Cedex 2, France. ${ }^{2}$ Departamento de

Matemática-CONICET, FCE, Univ. Austral, Rosario, Argentina.

\section{Publisher's Note}

Springer Nature remains neutral with regard to jurisdictional claims in published maps and institutional affiliations.

Received: 6 September 2017 Accepted: 1 March 2018 Published online: 12 March 2018

\section{References}

1. Du, Z: Singularly perturbed third-order boundary value problem for nonlinear systems. Appl. Math. Comput. 189, 869-877 (2007)

2. Du, Z., Ge, W., Zhou, M.: Singular perturbations for third-order nonlinear multi-point boundary value problem. J. Differ. Equ. 218, 69-90 (2005)

3. Lin, X.: Singular perturbations of third-order nonlinear differential equations with full nonlinear boundary conditions. Appl. Math. Comput. 224, 88-95 (2013)

4. Benbouziane, Z., Boucherif, A., Bouguima, S.M.: Existence result for impulsive third order periodic boundary value problems. Appl. Math. Comput. 206, $728-737$ (2008)

5. Cheng, M.: Nagumo theorems of third-order singular nonlinear boundary value problems. Bound. Value Probl. 2015, $135(2015)$

6. Liu, Z., Ume, J.S., Anderson, D.R., Kang, S.M.: Twin monotone positive solutions to a singular nonlinear third-order differential equation. J. Math. Anal. Appl. 334, 299-313 (2007)

7. Zhang, P.: Iterative solutions of singular boundary value problems of third-order differential equation. Bound. Value Probl. 2011, Article ID 483057 (2011)

8. Du, Z., Ge, W., Lin, X.: Existence of solutions for a class of third-order nonlinear boundary value problems. J. Math. Anal. Appl. 294, 104-112 (2004)

9. Lin, X., Du, Z., Liu, W.: Uniqueness and existence results for a third-order nonlinear multi-point boundary value problem. Appl. Math. Comput. 205, 187-196 (2008)

10. Cheng, Z., Xin, Y.: Multiplicity results for variable-coefficient singular third-order differential equation with a parameter. Abstr. Appl. Anal. 2014, Article ID 527162 (2015)

11. Du, Z:: Existence and uniqueness results for third-order nonlinear differential systems. Appl. Math. Comput. 218 2981-2987 (2011)

12. Jiang, W., Li, F.: Several existence theorem of monotone positive solutions for third-order multi-point boundary value problems. Bound. Value Probl. 2007, Article ID 17951 (2007)

13. Alsulami, H.H., Ntouyas, S.K., Al-Mezel, S.A., Ahmad, B., Alsaedi, A.: A study of third-order single-valued and multi-valued problems with integral boundary conditions. Bound. Value Probl. 2015, 25 (2015) 
14. Boucherif, A., Bouguima, S.M., Al-Malki, N., Benbouziane, Z.: Third order differential equations with integral boundary conditions. Nonlinear Anal. 71, e1736-e1743 (2009)

15. Boucherif, A., Bouguima, S.M., Benbouziane, Z., Al-Malki, N.: Third order problems with nonlocal conditions of integral type. Bound. Value Probl. 2014, 137 (2014)

16. Graef, J.R., Kong, L.: Positive solutions for third order semipositone boundary value problems. Appl. Math. Lett. 22, 1154-1160 (2009)

17. Guo, Y., Liu, Y., Liang, Y.: Positive solutions for the third-order boundary value problems with the second derivatives. Bound. Value Probl. 2012, 34 (2012)

18. Pang, H., Xie, W., Cao, L.: Successive iteration and positive solutions for a third-order boundary value problem involving integral conditions. Bound. Value Probl. 2015, 139 (2015)

19. Sun, J.P., Li, H.B.: Monotone positive solution of nonlinear third-order BVP with integral boundary conditions. Bound. Value Probl. 2010, Article ID 874959 (2010)

20. Wang, Y., Ge, W.: Existence of solutions for a third order differential equation with integral boundary conditions. Comput. Math. Appl. 53, 144-154 (2007)

21. Zhang, H.E.: Muliple positive solutions of nonlinear BVPs for differential systems involving integral conditions. Bound. Value Probl. 2014, 61 (2014)

22. Cannon, J.R., Lin, Y.: A Galerkin procedure for diffusion equations with boundary integral conditions. Int. J. Eng. Sci. 28(7), 579-587 (1990)

23. Dai, D.Q., Huang, Y.: Remarks on a semilinear heat equation with integral boundary conditions. Nonlinear Anal. 67, 468-475 (2007)

24. Dehghan, M.: Efficient techniques for the second-order parabolic equation subject to nonlocal specifications. Appl. Numer. Math. 52, 39-62 (2005)

25. Dehghan, M.: The one-dimensional heat equation subject to a boundary integral specifications. Chaos Solitons Fractals 32, 661-675 (2007)

26. Hao, D.N., Thanh, P.X., Lesnic, D., Ivanchov, M.: Determination of a source in the heat equation from integral observations. J. Comput. Appl. Math. 264, 82-98 (2014)

27. Kanca, F: The inverse problem of the heat equation with periodic boundary and integral overdetermination conditions. J. Inequal. Appl. 2013, 108 (2013)

28. Liu, Y:: Numerical solution of the heat equation with nonlocal boundary conditions. J. Comput. Appl. Math. 110 115-127 (1999)

29. Martin-Vaquero, J.: Two-level forth-order explicit schemes for diffusion equations subject to boundary integral specifications. Chaos Solitons Fractals 42, 2364-2372 (2009)

30. Martin-Vaquero, J., Vigo-Aguiar, J.: A note on efficient techniques for the second-order parabolic equation subject to non-local conditions. Appl. Numer. Math. 59, 1258-1264 (2009)

31. Martin-Vaquero, J., Vigo-Aguiar, J.: On the numerical soliton of the heat conduction equations subject to nonlocal conditions. Appl. Numer. Math. 59, 2507-2514 (2009)

32. Merazga, N., Bouziani, A.: On a time-discretization method for a semilinear heat equation with purely integral conditions in a nonclassical function space. Nonlinear Anal. 66, 604-623 (2007)

33. Martin-Vaquero, J., Wade, B.A.: On efficient numerical methods for an initial-boundary value problem with nonlocal boundary conditions. Appl. Math. Model. 36, 3411-3418 (2012)

34. Boucherif, A.: Second-order boundary value problems with integral boundary conditions. Nonlinear Anal. 70 , 364-371 (2009)

35. Liu, B., Li, J., Liu, L.: Nontrivial solutions for a boundary value problem with integral boundary conditions. Bound. Value Probl. 2014, 15 (2014)

36. Luand, G., Feng, M.: Positive Green's function and triple positive solutions of a second-order impulsive differential equation with integral boundary conditions and delayed argument. Bound. Value Probl. 2016, 88 (2016)

37. Tong, Z., Ding, W.: Existence of symmetric solutions for a class of BVP with integral boundary conditions. Bound. Value Probl. 2016, 84 (2016)

38. Zhang, X., Feng, M.: Positive solutions for a second-order differential equation with integral boundary conditions and deviating arguments. Bound. Value Probl. 2015, 222 (2015)

39. Zhang, X., Feng, M., Ge, W.: Existence result of second-order differential equations with integral boundary conditions and resonance. J. Math. Anal. Appl. 352, 311-319 (2009)

40. Zhang, L., Xuan, Z: Muliple positive solutions for a second-order boundary value problem with integral boundary conditions. Bound. Value Probl. 2016, 60 (2016)

41. Sun, M., Xing, Y.: Existence results for a king of forth-order impulsive integral boundary value problems. Bound. Value Probl. 2016, 81 (2016)

42. Zhang, X., Ge, W.: Positive solutions for a class of boundary-value problems with integral boundary conditions. Comput. Math. Appl. 58, 203-215 (2009)

43. Ji, Y., Guo, Y., Yao, Y.: Positive solutions for higher order differential equations with integral boundary conditions. Bound. Value Probl. 2015, 214 (2015)

44. Henderson, J., Luca, R.: Positive solutions for a system of semipositone coupled fractional boundary value problems. Bound. Value Probl. 2016, 61 (2016)

45. Li, H., Liu, L., Wu, Y.: Positive solutions for singular nonlinear fractional differential equation with integral boundary conditions. Bound. Value Probl. 2015, 232 (2015)

46. Wang, W., Guo, X.: Eigenvalue problem for fractional differential equations with nonlinear integral and disturbance parameter in boundary conditions. Bound. Value Probl. 2016, 42 (2016)

47. Berrone, L.R., Tarzia, D.A., Villa, L.T.: Asymptotic behavior of a non-classical heat conduction problem for a semi-infinite material. Math. Methods Appl. Sci. 23, 1161-1177 (2000)

48. Boukrouche, M., Tarzia, D.A.: Global solution to a non-classical heat problem in the semi-space $\mathbb{R}^{+} \times \mathbb{R}^{n-1}$. Q. Appl. Math. 72, 347-361 (2014)

49. Boukrouche, M., Tarzia, D.A.: A nonclassical heat conduction problem with non local source. Bound. Value Probl. 2017, 51 (2017) 
50. Ceretani, A.N., Tarzia, D.A., Villa, L.T.: Explicit solutions for a non-classical heat conduction problem for a semi-infinite strip with a non-uniform heat source. Bound. Value Probl. 2015, 156 (2015)

51. Salva, N.N., Tarzia, D.A., Villa, L.T: An initial-boundary value problem for the one-dimensional non-classical heat equation in a slab. Bound. Value Probl. 2011, 4 (2011)

52. Tarzia, D.A., Villa, L.T.: Some nonlinear heat conduction problems for a semi-infinite strip with a non-uniform heat source. Rev. Unión Mat. Argent. 41, 99-114 (1998)

53. Villa, L.T.: Problemas de control para una ecuación unidimensional no homogénea del calor. Rev. Unión Mat. Argent. 32, 163-169 (1986)

54. Miller, R.K.: Nonlinear Volterra Integral Equations. Benjamin, Elmsford (1971)

55. Adomian, G.: Solving Frontier Problems of Physics Decomposition Method. Springer, Berlin (1994)

56. Wazwaz, A.M.: Linear and Nonlinear Integral Equations. Methods and Applications. Springer, Heidelberg (2011)

\section{Submit your manuscript to a SpringerOpen ${ }^{\circ}$} journal and benefit from:

- Convenient online submission

- Rigorous peer review

- Open access: articles freely available online

- High visibility within the field

- Retaining the copyright to your article

Submit your next manuscript at $\gg$ springeropen.com 